**THIS IS A PRE-PRINT. THE UPDATED, PEER-REVIEWED VERSION OF THIS

PAPER IS NOW PUBLISHED IN EUROPEAN JOURNAL OF TRAUMA AND

DISSOCIATION (04/08/2021)

https://doi.org/10.1016/j.ejtd.2021.100219

\title{
Traumatic Impact of the COVID-19 Pandemic in an International Sample: Contribution of Fatalism to Psychological Distress and Behavior Change
}

\author{
Olga Bogolyubova ${ }^{1}$, Andrea Sainz-Maza Fernandez ${ }^{2}$, Belen Tristan Lopez ${ }^{2}$, Pamela Portelli ${ }^{1}$
}

Author Note

Olga Bogolyubova, ${ }^{1}$ Department of Psychology, Faculty of Social Wellbeing, University of Malta, Tal-Qroqq, Msida, MSD 2080, Malta; email: olga.bogolyubova@um.edu.mt

Andrea Sainz-Maza Fernandez, ${ }^{2}$ Faculty of Psychology, University of Almería, San Urbano Road, s/n, 04120 La Cañada, Almería, España; email: asf227@inlumine.ual.es

Belen Tristan Lopez, ${ }^{2}$ Faculty of Psychology, University of Almería, San Urbano Road, s/n, 04120 La Cañada, Almería, España; email: btl184@inlumine.ual.es

Pamela Portelli, ${ }^{1}$ Department of Psychology, Faculty of Social Wellbeing, University of Malta, Tal-Qroqq, Msida, MSD 2080, Malta; email: pport03@um.edu.mt

Correspondence concerning this article should be addressed to Olga Bogolyubova, ${ }^{1}$ Department of Psychology, Faculty of Social Wellbeing, University of Malta, Tal-Qroqq, Msida, MSD 2080, Malta; email: olga.bogolyubova@um.edu.mt

Funding: none

Conflicts of interest: none. 


\begin{abstract}
This study aimed to assess the traumatic impact of the COVID-19 outbreak and to explore the contribution of fatalism to a) psychological distress and b) pandemic-related behavior change. Data for the study were collected via an anonymous online survey. The survey included questions about demographic characteristics, health status, pandemic-related stress, impact of COVID-19 on health and behavior, posttraumatic stress symptoms (PTSS), and fatalism. The sample included 465 participants from 30 countries (mean age $=36.87, \mathrm{SD}=13.39$, age range $=$ $18-76 ; 80 \%$ female). While none of the study participants have been diagnosed with COVID19, almost $25 \%$ knew someone who had received the diagnosis. The overwhelming majority (79\%) had to make changes to their daily routine, and $48.82 \%$ of the sample reported moderate to severe PTSS. Fatalism was found to be a statistically significant predictor of PTSS in a multiple regression model and was also associated with lack of behavior changes in response to the pandemic. Our findings indicate that the COVID-19 pandemic is a traumatic event with a potential to affect mental health and well-being of individuals and communities. Fatalism may contribute to severity of psychological distress and diminish individuals' ability to engage in health-protective behaviors.
\end{abstract}

Keywords: COVID-19, coronavirus, posttraumatic stress symptoms, fatalism, health behavior 


\section{Traumatic Impact of the COVID-19 Pandemic in an International Sample: Contribution of Fatalism to Psychological Distress and Behavior Change}

The world is currently in the midst of the COVID-19 pandemic. Following an initial outbreak in China in December 2019, the novel coronavirus has spread across the globe. As of July 24, 2020, the number of deaths worldwide has exceeded 619000 cases, with over 15 million people infected in 216 countries (World Health Organization, 2020). The virus has a fast transmissibility and is highly contagious, posing serious threats to public health.

On the global level, COVID-19 has significantly slowed down local economies, disrupting world trade and movement, resulting in significant losses for national and international businesses (Haleem \& Javaid, 2020). On the individual level, it has affected the daily lives and well-being by escalating social isolation and economic hardships, by disrupting routines, and, in some cases, by causing loss of health and/or bereavement.

Both historically and in the present times, pandemics have resulted in loss of lives and in severe social and economic disruptions. They present significant challenges for the medical profession in terms of containment and treatment. Emerging evidence suggests that infectious disease outbreaks also have significant effect on the mental health and psychological functioning of affected populations.

Research into the psychological effects of the most recent pandemics, caused by SARS, MERS, Ebola, and Zika viruses, indicates that survivors, family members, health care professionals, and members of the general public can be significantly affected. Fear-related behaviors were found to have contributed significantly to the course of Ebola outbreak in West Africa and its survivors experienced significant psychological distress and symptoms of mental 
disorders such as PTSD (Hugo et al., 2015, Mohammed et al., 2015, Shultz et al., 2016). Zika virus has been associated with negative consequences for maternal mental health (Tucci et al., 2017). A study conducted among SARS survivors in Hong Kong (Cheng, Wong, Tsang \& Wong, 2004) found significant levels of anxiety and depression symptoms at 1-month post recovery. Subsequently, in a cohort study of SARS survivors, at 30-month follow-up PTSD was found to be the most prevalent psychiatric complication, followed by depression (Mak et al., 2009).

Not only survivors are affected. A number of studies conducted with healthcare workers in the wake of the SARS epidemic have demonstrated heightened concern for personal and family health, significant increase in emotional distress and psychiatric morbidity, as well as an escalation of anxiety and uncertainty brought about by fears of SARS contagion, stigmatization, and loneliness (Maunder et al., 2003, Nickell et al., 2004). Similar findings were described in studies assessing the impact of MERS epidemic on the mental health of health care providers, with fears for personal safety and symptoms of posttraumatic stress reported (Lee et al., 2018, Khalid et al., 2016). Among the general public, high prevalence of helplessness and fear responses was registered, with more than $15 \%$ exhibiting symptoms of posttraumatic stress in response to the SARS outbreak (Lau et al., 2005). In healthy individuals who had been quarantined due to exposure to MERS, symptoms of anxiety and feelings of anger were reported (Jeong et al., 2016).

When it comes to the current COVID-19 pandemic, research into its psychological impact is still in its early stages. While in recent months the amount of publications on the subject has been rapidly increasing, data on the effects of the pandemic on the mental health and 
well-being of the general public is still limited (Vindegaard \& Benros, 2020). Available studies demonstrate the following results:

In a nationwide, large-scale study of mental health symptoms during the pandemic in China's general population, $35 \%$ of the respondents reported significant psychological distress (Qiu et al., 2020), and in a study by Sonderskov et al. (2020) a decline in subjective well-being scores was recorded in a representative sample of Danish population (in comparison with prepandemic scores).

A number of conducted studies focused specifically on the traumatic impact of the pandemic. For instance, in three different studies conducted in China, the prevalence of posttraumatic stress symptoms (ranging from $4.6 \%$ to $53.8 \%$ depending on the methodology utilized) was assessed along with the demographic and behavioral predictors of elevated distress (Liu et al., 2020, Sun et al., 2020, Wang et al., 2020). In Italy, 37\% of the respondents reported PTSS symptoms (Rossi et al., 2020), and in a Lebanese study on the effects of COVID-19 quarantine, a significant proportion of the respondents reported experiencing similar symptoms (Fawaz \& Samaha, 2020).

When it comes to infectious disease outbreaks, their trajectory and the success of containment efforts largely depends on the behavior of individuals and their ability to adopt health-protective behaviors and follow the guidelines issued by the health authorities (Ibuka et al., 2010). Existing research from prior similar epidemics demonstrates that the likelihood of adopting health-protective behaviors is impacted by risk perceptions, as demonstrated by Rubin et al. (2009) in a study conducted during the swine flu outbreak; perceived efficacy of precautionary behaviors and level of anxiety as shown by Lau et al. (2010) during the H1N1 influenza epidemic. Emerging research on health-protective behaviors during the COVID-19 
pandemic indicates that perceived credibility of received information (Lep, Babnik \& Hacin Beyazoglu, 2020) and fear of COVID-19 (Harper, Satchell, Fido \& Latzman, 2020) predictive engagement in health-protective behaviors.

At the moment, our understanding of the individual-level predictors of both psychological distress and behavior change (or lack thereof) in the context of COVID-19 pandemic is still limited. We hypothesize that fatalism can play an important role in shaping the psychological response in the situation of a disease outbreak by contributing both to the development of greater psychological distress and to the diminished ability to engage in healthprotective behaviors.

The term 'fatalism' is grounded within the generalized expectancy framework. It refers to an attitude of resignation and the belief that one is powerless in the face of inevitable life events (Abraído-Lanza et al, 2007). Individuals with a high level of fatalism are less likely to cope with stress, tend to adopt a passive attitude, and may experience suicidal ideation (Laghi, Baiocco \& D' Alessi, 2009). In terms of mental health outcomes, having fatalistic beliefs has been found to increase the risk of developing depression and post-traumatic stress disorder (Maercker, BenEzra, Esparza \& Augsburger, 2019; Piña-Watson \& Abraído-Lanza, 2017). When it comes to health behaviors, fatalism has been associated with elevated risk of HIV infection (Kalichman et al., 1997, Sileo et al., 2019) and has been described as a barrier to cancer screening (Espinosa de los Monterros \& Gallo, 2010). It has also been identified as a factor in non-completion of HPV vaccination (Vanderpool et al., 2015).

Our research study pursued two objectives: a) to expand the understanding of the psychological impact of COVID-19 pandemic as a traumatic event by assessing the prevalence of subjective stress and posttraumatic stress symptoms (PTSS) during the outbreak in an 
international sample and b) to explore the contribution of fatalism to pandemic-related behavior change (Hypothesis 1) and to psychological distress symptoms (Hypothesis 2).

\section{Method}

\section{Participants and Procedures}

Data collection for this study was conducted between April 9 and May 5, 2020 by means of an anonymous online survey targeting an international convenience sample of adults. The link to the study was posted on Reddit threads dedicated to participant recruitment and on social media. Snowballing approach was taken, and the participants were encouraged to share the link to the study with other people. The inclusion criteria, indicated in the Informed Consent form, were being 18 or older and fluency in English (language of the survey). This research was conducted in conformity with [MASKED FOR PEER REVIEW] Research Ethics Review Procedures.

\section{Measures}

Demographic Information. Age, gender, marital status, household composition (number of people in the household), parental status, level of education, employment status, and country of residence were assessed.

Subjective Health Status was assessed by a single question adapted from a World Values Survey questionnaire (Adesanya, Rojas, Darboe \& Beogo, 2017): “All in all, how would you describe your state of health these days?" Response options were: very good, good, fair, or poor.

COVID-19 Impact. This section of the survey included the following four questions: “Have you been diagnosed with COVID-19?” (response options: yes, no, prefer not to say); "Has anyone you know personally (e.g. family member, friend, colleague etc.) been diagnosed with COVID-19?" (response options: yes, no, don't know, prefer not to say); "Have you had to 
remain in quarantine due to contact with someone infected with COVID-19 or due to having traveled abroad?" (response options: yes, no, prefer not to say); "Have you had to modify your behavior (e.g. self-isolate, work from home, changes in eating habits etc.) as a result of the COVID-19 pandemic?" (response options: no, not at all; I have made some minor changes; I had to make some significant changes to my daily routine; My daily life has been changed entirely). COVID-19 Stress. This section of the survey included one question aimed at gauging the overall subjective level of stress related to the pandemic. Participants were asked: "Overall, how would you assess your level of stress in relation to COVID-19 pandemic?" This was followed up by four questions assessing the level of subjective stress in relation to different domains of possible concerns. These were concerns for own health, concerns for the health of loved ones, decreased financial security, decreased job security, and social isolation. All of these questions had five response levels: $1=$ not at all stressed, $2=$ mildly stressed, $3=$ moderately stressed, $4=$ very stressed, 5 extremely stressed.

Impact of Events Scale-Revised (IES-R) was used to assess the traumatic impact of the pandemic and to evaluate the prevalence of PTSS in the study sample. IES-R (Weiss \& Marmar, 1997) is a well-established measure used to assess the psychological impact of potentially traumatic events. It consists of 22 items measured on a 5-point Likert scale and includes three subscales (Intrusion, Avoidance, Hyperarousal). While IES-R does not align with the updated DSM 5 criteria for PTSD and can no longer be used as a diagnostic tool for PTSD, it remains a valid screening instrument for posttraumatic stress symptoms in non-clinical populations (Hosey et al., 2019). For the purposes of this study, item 14 ("I found myself acting or feeling like I was back at that time.") was removed as it didn't comply with assessing the impact of an ongoing situation. The resulting version of IES-R with 21 items demonstrated good internal consistency 
with a Cronbach's $\alpha=.93$ for the total score and for each of the subscales (Intrusion: Cronbach's $\alpha=.89$; Avoidance: Cronbach’s $\alpha=.82$; Hyperarousal: Cronbach’s $\alpha=.83$ ).

Fatalism was assessed using a 6-item fatalism subscale of a larger "Multidimensional Fatalism Measure" (Esparza et al., 2015) following the approach described in Maercker et al. (2019). In our study, the scale demonstrated good internal consistency with Cronbach's $\alpha=.87$ (for the total score).

\section{Data Quality Control.}

Concerns exist in relation to the quality of the data collected by means of anonymous online surveys; these relate both to the possibility of bots "participating" and filling the survey with random responses and to the possible malicious and/or inattentive behaviors of human subjects resulting in meaningless response patterns. A number of solutions have been proposed to mitigate these treats to the integrity of the data and assure the quality of collected responses (Daniel et al., 2018). The following data control and cleansing methods were used in our study.

First, the survey included two questions (age and country of residence) where the response had to be typed by the participants. If both of these fields were left blank, the responses were removed from the data set as the absence of both hand-typed answers could be indicative of a bot or a human participant with low motivation and/or nonchalant attitude.

Second, a control question was included in the survey. It was presented to participants as follows: "If you answer this question, we will not count your entry, because you are either not paying attention or you are a spam robot." The response options were: yes, no, prefer not to say. The correct response was to leave this question unanswered. The responses of participants who did not leave this question blank were removed from the survey as this could be indicative of a bot, a human participant "answering" the questions without actually reading them, or of a human 
participant whose command of English was, in fact, not sufficient to comprehend the questionnaire and provide reliable responses.

Finally, the data were checked for straight-line/pattern responses. None were identified.

\section{Data Analysis}

Statistical analysis was conducted using IBM SPSS 26 for Windows. Descriptive statistics were calculated for demographic characteristics, self-reported health status, pandemicrelated concerns, and the two psychometric measures (IES-R and fatalism scores). Spearman's correlation coefficient and Mann Whitney U were employed to explore possible associations between demographic characteristics (age, gender) and the subjective impact of COVID-19 pandemic. A one-way ANOVA was conducted to assess if the fatalism score varied between groups with different levels of behavior modification during the pandemic. A standard multiple regression was used to identify the predictors of posttraumatic stress symptoms in the study sample. No imputation was used for missing data.

\section{Results.}

\section{Study Sample}

During the data collection period, 504 responses to the survey were recorded. Of these, 33 were removed from the dataset due to the failure to respond to the control question correctly. Another two responses were removed due to the missing responses to both age and country of residence questions. Finally, four entries were removed because, while the informed consent form clearly indicated that the survey is open only to individuals aged 18 and over, the indicated age in these four cases was lower than 18. No entries with suspicious patterns of responses were identified. 
After all of the data cleaning procedures were completed, the final sample included 465 participants residing in 30 different countries. Countries with the biggest representation in the sample were as follows: $47.1 \%$ of the participants came from Malta, $11.4 \%$ from Russia; $10.5 \%$ from the USA, and $10.3 \%$ from Spain. The full list of countries of residence indicated by the study participants can be found in Table 1 .

[Table 1 goes here]

The mean age of the study sample was $36.87(\mathrm{SD}=13.39$; age range $=18-76)$. Of all the participants, 30 (6.5\% of the sample) did not indicate their age.

The majority of participants $(80 \% ; \mathrm{n}=372)$ were female and $18.7 \%(\mathrm{n}=87)$ identified as male. Two participants identified as non-binary; one participant indicated "Other" as a response option; one participant chose the response option "Prefer not to say," and two participants chose not to respond to this question.

The majority of the sample $(67.3 \%)$ were in possession of a university degree; $47 \%$ were employed full time and $21.5 \%$ were students; $46.1 \%$ were married and $32 \%$ were single (never married); $66.2 \%$ were childless and $31.4 \%$ had children in the household; almost $11 \%$ reported living alone. The detailed demographic description of the sample is presented in Table 2.

[Table 2 goes here]

The majority of the sample assessed their health as good or very good (84.3\%). Slightly over $14 \%(\mathrm{n}=66)$ described their health as fair, and seven participants $(1.5 \%)$ stated that their health was poor.

\section{Impact of COVID-19 on health and behavior}


None of the participants reported having been diagnosed with COVID-19. The majority of participants $(68.6 \%)$ did not personally know anyone who has been diagnosed with COVID; $23.7 \%$ knew someone who has been diagnosed, and $7.7 \%$ reported that they were not sure.

Experience of quarantine (either due to international travel or exposure to the virus) was reported by $9 \%(n=42)$ of the total number of study participants.

The majority of participants $(79 \%)$ reported that they have had to modify their life style significantly in response to the pandemic. Slightly over $18 \%$ of the participants reported having made minor changes to their daily living, and only $3 \%$ of the participants reported that they have made no changes at all.

\section{Stress related to the COVID-19 pandemic}

In terms of overall self-assessment of stress related to the pandemic, high levels of stress were reported by $18.7 \%$ of the sample. By areas of concern, high level of stress ("very stressed" and "extremely stressed" response options) in relation to personal health was reported by $28 \%$ of the sample; by over $70 \%$ in relation to health of loved ones; by $35.5 \%$ in relation to decreased financial security; by $30.4 \%$ in relation to decreased job security, and by over $44 \%$ in relation to social isolation. For more detail see Table 3.

[Table 3 goes here]

\section{Demographic characteristics and the impact of COVID-19 pandemic}

There were negative correlations (Bonferroni corrected) between age and the following areas of pandemic-related concerns: decreased financial security $\left(r_{s}=-.215, p<.0005\right)$, decreased job security $\left(\mathrm{r}_{\mathrm{s}}=-.316, \mathrm{p}<.0005\right)$, and social isolation $\left(\mathrm{r}_{\mathrm{s}}=-.192, \mathrm{p}<.0005\right)$.

Gender differences in the impact of the pandemic were assessed for the study participants who have identified as either male or female $(n=459)$ as there were too few members of other 
groups. A series of Mann-Whitney U tests were run to determine if there were differences in the impact of the pandemic between male and female participants. Scores for the overall selfassessment of stress in males $(M=2.41)$ and in females $(M=2.78)$ were statistically significantly different, $U=12953, z=-3.050, \mathrm{p}<.005$. Statistically significant differences were also found in scores for concerns related to personal health $\left(\mathrm{M}_{\text {males }}=2.39, \mathrm{M}_{\text {female }}=2.97, U=\right.$ $11420, z=-4.429, \mathrm{p}<.0005)$; concerns for the health of loved ones $\left(\mathrm{M}_{\text {males }}=3.57, \mathrm{M}_{\mathrm{female}}=\right.$ 4.04, $U=11975, z=-3.941, \mathrm{p}<.0005)$; and concerns related to social isolation $\left(\mathrm{M}_{\text {males }}=2.89\right.$, $\left.\mathrm{M}_{\text {female }}=3.27, U=13528, z=-2.441, \mathrm{p}<.0005\right)$.

\section{Posttraumatic stress symptoms in the study sample}

The mean total IES-R score in the study sample was $30.2($ range $=0-73 ; \mathrm{SD}=16.53)$. Of the total number of the study participants, 33.3\% $(\mathrm{n}=155)$ reported minimal psychological impact of COVID-19 pandemic with IES-R total score $\leq 23$; mild psychological impact was registered in $17.85 \%(n=83)$ with a score between 24 and 32 , and $48.82 \%(n=227)$ reported moderate to severe psychological impact with a score of $\geq 33$. The scores for the three subscales were as follows: Intrusion $(\mathrm{M}=1.48, \mathrm{SD}=.95 ;$ range $=0-4)$, Avoidance $(\mathrm{M}=1.41, \mathrm{SD}=.79$; range $=0-3.38)$, Hyperarousal $(\mathrm{M}=1.42, \mathrm{SD}=.95 ;$ range $=0-4)$.

\section{Fatalism and behavior change in response to pandemic}

A one-way ANOVA was conducted to determine if the level of fatalism varied between groups with different levels of behavior change in response to COVID-19 pandemic. For this analysis, participants were classified into three groups: "made no changes at all or made only minor changes" $(n=97)$, "made significant changes" $(n=203)$, "lifestyle changed entirely" $(n=$ 163). There were no outliers, data was normally distributed for each group as assessed by the visual inspection of Q-Q plots, and there was homogeneity of variances as assessed by Levene's 
test of homogeneity of variances $(p=.608)$. The difference in fatalism score was statistically significant between groups with different levels of behavior change, $F(2,460)=5.254$, p. $<.01$, $\eta^{2}=.02$. Fatalism score in the "made no changes at all or made only minor changes" group $(\mathrm{M}=$ $19.75, \mathrm{SD}=6.14)$ was higher than that in the "made significant changes" group $(\mathrm{M}=17.4, \mathrm{SD}=$ 5.95) and in the "lifestyle changed entirely" group $(\mathrm{M}=17.9, \mathrm{SD}=5.95)$. Tukey-Kramer post hoc analysis demonstrated that the mean increase in fatalism score from "made no changes at all or made only minor changes" group to "made significant changes" group $(2.35,95 \%$ CI [.63, 4.1]) was statistically significant with $\mathrm{p}<.005$, as well as the increase "made no changes at all or made only minor changes" group to "lifestyle changed entirely" group $(1.86,95 \%$ CI [.07, 3.65], $\mathrm{p}<.05)$. The difference in fatalism between "made significant changes" and lifestyle changed entirely" groups was not statistically significant.

\section{Predictors of posttraumatic stress symptoms}

A multiple regression was run to predict PTSS symptoms (as measured by IES-R total score) in response to COVID-19 pandemic. Three dummy variables were created to represent the variable derived from a question "Has anyone you know personally (e.g. family member, friend, colleague, etc.) been diagnosed with COVID-19?" with three response options. Marital status and parental status questions were recoded as binary nominal variables. The assumptions of normality, linearity, multicollinearity, and homoscedacity were not violated. The model was statistically significant with $F(18,380)=25.427, \mathrm{p}<.0005$. Overall level of subjective stress (by self-assessment), concerns for personal health, concerns related to diminished job security, concerns related to social isolation, and fatalism were statistically significant predictors. Adjusted $R^{2}$ for the model was 0.53 . See Table 4 for more detail.

[Table 4 goes here] 


\section{Discussion}

The study reported in this paper pursued two main objectives: a) to assess the traumatic impact of the COVID-19 outbreak in an international convenience sample and b) to explore the contribution of fatalism to behavior change and to psychological distress in response to the pandemic.

Our findings indicate that the study participants were significantly affected by the pandemic. While none of the study participants have been diagnosed with COVID-19, almost $25 \%$ knew someone who had received the diagnosis. The overwhelming majority had to make significant changes to their daily routine as a result of the pandemic, and nearly $20 \%$ of the sample reported high level of COVID-related stress. Concerns for the health of loved ones was the most prominent source of stress with $70 \%$ of the respondents reporting high and very high levels of stress in relation to this. This is in similarity to the results reported in a study conducted in Chinese general public, where over $75 \%$ of the participants reported worrying about family members getting infected (Wang et al., 2020).

When it comes to the demographic differences in the subjective response to the situation of the pandemic, older participants were less likely to report experiencing stress related to the decreased financial and job security which may be associated with having more established careers or no longer being active in the job market. Interestingly, older participants also reported less stress in relation to social isolation; however, for this association the effect size was small.

Male participants reported lower overall stress, as well as lower levels of stress related to own health, health of loved ones, and social isolation. This may be indicative of gender differences in stress response where women tend to report higher levels of subjective distress 
(Goldfarb, Seo \& Sinha, 2019), as well as of the gender role expectations which may result in men being less likely to report stress associated with personal vulnerability and/or relationships.

The traumatic impact of the pandemic, as measured by the IES-R total score, was found to be significant with almost half of the sample reporting moderate to severe psychological impact as indicated by a score of $\geq 33$, a cut-off point proposed in Creamer, Bell \& Failla (2004). This result is in line with other studies of COVID-related posttraumatic response. For instance, in a study by Wang et al. (2020) 53.8\% of the respondents scored above the indicated cut-off point.

In the multiple regression model, the overall level of subjective stress, as well as concerns for personal health, concerns related to diminished job security, stress related to social isolation, and fatalism were found to predict PTSS symptoms in the study sample; however, none of the demographic characteristics included in the model made a statistically significant contribution. Overall, the model explained $53 \%$ of the variance of the outcome variable, which is indicative of a large effect size according to Cohen (1988).

In relation to demographics, our results differ from a number of recent studies where female gender and, in some instances, older age, were found to be associated with higher levels of COVID-related PTSS (Liu et al., 2020, Qiu et al., 2020, Rossi et al., 2020, Sun et al., 2020, Wang et al., 2020). However, our findings in this respect are similar to the ones reported by Fawaz et al. (2020) where no gender differences in posttraumatic symptomatology were found in a Lebanese sample.

When it comes to our findings regarding fatalism as a predictor of COVID-related distress, the results are partially in line with a recent study by Hayes et al. (2020) where higher levels of fatalism were associated with greater psychological distress as manifested by depressive affect. However, it must be noted that in the same study higher level of fatalism was associated 
with lower levels of experienced fear, which, to an extent, can be seen as a contrast to our findings, if symptoms of posttraumatic stress are conceptualized as fear-related psychopathology.

Finally, an association between fatalism and the extent of behavioral changes was identified. Study participants with higher levels of fatalism were less likely to report having changed their behavior in response to the pandemic. This is consistent with the two recent studies on the association between fatalism and intentions to perform COVID-related preventive behaviors such as social distancing and handwashing. In a previously mentioned study by Hayes et al. (2020), an association between higher levels of fatalism and lower level of behavioral intentions to support prevention efforts was found, and in a study by Jimenez et al. (2020) fatalism, conceptualized as associating coronavirus with death, negatively predicted intentions to modify behavior.

To summarize, the study results presented in this paper make a contribution to the growing body of research on the psychological impact of the COVID-19 pandemic. Both of the proposed hypotheses were supported by the data analyses identifying fatalism as a statistically significant predictor of PTSS and as a factor in lack of behavior changes in response to the pandemic.

\section{Limitations}

The study had several limitations that need to be taken into account. The study was based on self-report and employed a convenience sample. While the study participants came from a wide range of countries, small number of participants from the majority of them did not allow for cross-country comparisons. Moreover, the sample was overwhelmingly female and in possession of a university degree. This may impede the generalization of findings to other demographics or social groups. No information about the participants' mental health history was collected. 
Participants experiencing psychological problems or traumatic events prior to the pandemic may have been more likely to report PTSS in this study. In addition, as this was a correlational study and the questionnaire did not specify if the lack of behavior changes was voluntary or not, it is impossible to determine the direction of the relationship between fatalism and behavior modification - it is not clear if higher levels of fatalism led to the unwillingness to modify behavior or if being unable to modify behavior (e.g. due to professional occupation) led to the endorsement of fatalistic beliefs.

\section{Conclusion}

The results of our study indicate that the outbreak of COVID-19 has resulted in psychological distress and can be considered a significant traumatic event. This will likely result in increased demand for psychological services and interventions in the aftermath of the pandemic and mental health services should be scaled up to address the needs of affected populations. It would also be useful to monitor vulnerable individuals, such as those suffering from PTSS during the peak of the pandemic, to prevent further deterioration of their mental health. Health care professionals and policy makers should also be aware of the role that mental health symptoms and psychological characteristics (such as fatalism) may play in the individuals' ability to follow public health recommendations.

\section{Declaration of Interest Statement}

Authors declare no conflict of interest. 


\section{References}

Abraído-Lanza, A. F., Flórez, K. R., Céspedes, A., Aguirre, A. N., De La Cruz, A. A. \& Viladrich, A. (2007). Commentary: Fatalismo reconsidered: A cautionary note for health-related research and practice with Latino populations. Ethnicity and Disease, 17(1), 153-158.

Adesanya, A. O., Rojas, B. M., Darboe, A. \& Beogo, I. (2017). Socioeconomic differential in selfassessment of health and happiness in 5 African countries: Finding from World Value Survey. PLoS ONE, 12(11), 1-12. doi:10.1371/journal.pone.0188281

Cheng, S., Wong, C., Tsang, J., \& Wong, K. (2004). Psychological distress and negative appraisals in survivors of severe acute respiratory syndrome (SARS). Psychological Medicine, 34(7), 11871195. doi:10.1017/S0033291704002272

Creamer, M., Bell, R., \& Failla, S. (2003). Psychometric properties of the Impact of Event ScaleRevised, Behaviour Research and Therapy, 41(12), 1489-1496. doi:10.1016/j.ynstr.2019.100177

Daniel, F., Kucherbaev, P., Cappiello, C., Benatallah, B. \& Allahbakhsh, M. (2018). Quality control in crowdsourcing: A survey of quality attributes, assessment techniques, and assurance actions. ACM Computing Surveys. Association for Computing Machinery, 51(1) 7-40. doi: $10.1145 / 3148148$

De Los Monteros, K. E. \& Gallo, L. C. (2011). The relevance of fatalism in the study of Latinas' cancer screening behavior: A systematic review of the literature. International Journal of Behavioral Medicine, 18, 310-318. doi: 10.1007/s12529-010-9119-4

Esparza, O. A., Wiebe, J. S. \& Quiñones, J. (2015). Simultaneous development of a Multidimensional Fatalism Measure in English and Spanish. Current Psychology, 34(4), 597612. doi:10.1007/s12144-014-9272-z 
Fawaz, M. \& Samaha, A. (2020). COVID-19 quarantine: Post-traumatic stress symptomatology among Lebanese citizens. International Journal of Social Psychiatry, 00(0), 1-9. doi: $10.1177 / 0020764020932207$

Goldfarb, E. V., Seo, D. \& Sinha, R. (2019). Sex differences in neural stress responses and correlation with subjective stress and stress regulation. Neurobiology of Stress, 11, 1-10.

Harper, C.A., Satchell, L.P., Fido, D. et al. Functional Fear Predicts Public Health Compliance in the COVID-19 Pandemic. Int J Ment Health Addiction (2020). https://doi.org/10.1007/s11469020-00281-5

Hayes, J. (2020). Fatalism in the fight against COVID-19: Implications for mitigation and mental health. Researchgate Net, 1-33. doi:10.31219/osf.io/t6zmv

Hosey, M. M., Bienvenu, O. J., Dinglas, V. D., Turnbull, A. E., Parker, A. M., Hopkins, R. O., Neufeld, K. J. \& Needham, D. M. (2019). The IES-R remains a core outcome measure for PTSD in critical illness survivorship research. Critical Care, 23(1), 362. doi:10.1186/s13054-019-26303

Hugo, M., Declerck, H., Fitzpatrick, G., Severy, N., Gbabai, O. B.-M., Decroo, T. \& Van Herp, M. (2015). Post-traumatic stress reactions in Ebola virus disease survivors in Sierra Leone. Emergency Medicine: Open Access, 05(6), 1-4. doi:10.4172/21657548.1000285

Ibuka, Y., Chapman, G.B., Meyers, L.A. et al. The dynamics of risk perceptions and precautionary behavior in response to 2009 (H1N1) pandemic influenza. BMC Infect Dis 10, 296 (2010). https://doi.org/10.1186/1471-2334-10-296 
Jeong, H., Yim, H. W., Song, Y. J., Ki, M., Min, J. A., Cho, J., \& Chae, J. H. (2016). Mental health status of people isolated due to Middle East Respiratory Syndrome. Epidemiology and Health, 38, e2016048. https://doi.org/10.4178/epih.e2016048

Jimenez, T., Restar, A., Helm, P. J., Cross, R. I., Barath, D. \& Arndt, J. (2020). Fatalism in the context of COVID-19: Perceiving coronavirus as a death sentence predicts reluctance to perform recommended preventive behaviors. SSM - Population Health, 11, 1-6. doi:10.1016/j.ssmph.2020.100615

Kalichman, S. C., Kelly, J. A., Morgan, M., \& Rompa, D. (1997). Fatalism, current life satisfaction, and risk for HIV infection among gay and bisexual men. Journal of Consulting and Clinical Psychology, 65(4), 542-546. doi:10.1037/0022-006X.65.4.542

Khalid, I., Khalid, T. J., Qabajah, M. R., Barnard, A. G. \& Qushmaq, I. A. (2016). Healthcare workers emotions, perceived stressors and coping strategies during a MERS-CoV outbreak. Clinical Medicine and Research, 14(1), 7-14. doi:10.3121/cmr.2016.1303

Laghi, F., Baiocco, R., D’Alessio, M. \& Gurrieri, G. (2009). Suicidal ideation and time perspective in high school students. European Psychiatry, 24(1), 41-46. doi:10.1016/j.eurpsy.2008.08.006

Lau, J. T. F, Griffiths, S., Choi, K. - C., Lin, C. (2010). Prevalence of preventive behaviors and associated factors during early phase of the H1N1 influenza epidemic, American Journal of Infection Control, Volume 38, Issue 5, pp. 374-380, https://doi.org/10.1016/j.ajic.2010.03.002.

Lau, J. T. F., Yang, X., Pang, E., Tsui, H. Y., Wong, E. \& Yun, K. W. (2005). SARS-related perceptions in Hong Kong. Emerging Infectious Diseases, 11(3), 417-424. doi:10.3201/eid1103.040675 
Lee, S. M., Kang, W. S., Cho, A. R., Kim, T. \& Park, J. K. (2018). Psychological impact of the 2015 MERS outbreak on hospital workers and quarantined hemodialysis patients. Comprehensive Psychiatry, 87, 123-127. doi:10.1016/j.comppsych.2018.10.003

Lep, Z., Babnik, K., Hacin Beyazoglu, K. (2020). Emotional Responses and Self-Protective Behavior Within Days of the COVID-19 Outbreak: The Promoting Role of Information Credibility, Frontiers in Psychology, Volume 11, page 1846, DOI=10.3389/fpsyg.2020.01846

Li, S., Wang, Y., Xue, J., Zhao, N. \& Zhu, T. (2020). The impact of covid-19 epidemic declaration on psychological consequences: A study on active Weibo users. International Journal of Environmental Research and Public Health, 17(6), 1-9. doi:10.3390/ijerph17062032

Liu, N., Zhang, F., Wei, C., Jia, Y., Shang, Z., Sun, L., Wu, Y. L., Sun, Z. Zhou, Y., Wang, Y. \& Liu, W. (2020). Prevalence and predictors of PTSS during COVID-19 outbreak in China hardesthit areas: Gender differences matter. Psychiatry Research, 287, 1-7. doi:10.1016/j.psychres.2020.112921

Maercker, A., Ben-Ezra, M., Esparza, O. A. \& Augsburger, M. (2019). Fatalism as a traditional cultural belief potentially relevant to trauma sequelae: Measurement equivalence, extent and associations in six countries. European Journal of Psychotraumatology, 10(1), 1-13. doi:10.1080/20008198.2019.1657371

Mak, I. W. C., Chu, C. M., Pan, P. C., Yiu, M. G. C. \& Chan, V. L. (2009). Long-term psychiatric morbidities among SARS survivors. General Hospital Psychiatry, 31(4), 318-326. doi:10.1016/j.genhosppsych.2009.03.001 
Maunder, R., Hunter, J., Vincent, L., Bennett, J., Peladeau, N., Leszcz, M., Sadavoy, J., Verhaeghe, L., Steinberg, R. \& Mazzulli, T. (2003). The immediate psychological and occupational impact of the 2003 SARS outbreak in a teaching hospital. CMAJ, 168(10), 1245-1251.

Mohammed, A., Sheikh, T. L., Gidado, S., Poggensee, G., Nguku, P., Olayinka, A., Ohuabunwo, C., Ndadilnasiya, W., Shuaid, F., Adeyeni, J., Uzoma, O., Ahned, A., Doherty, F., Beysolow, S., Nzuki, C., Nasidi, A., Oyemakinde, A., Oguntimehin, O., Adeshina, I. \& Obiako, R. O. (2015). An evaluation of psychological distress and social support of survivors and contacts of Ebola virus disease infection and their relatives in Lagos, Nigeria: A cross sectional study - 2014. BMC Public Health, 15(1), 1-8. doi:10.1186/s12889-015-2167-6

Nickell, L. A., Crighton, E. J., Tracy, C. S., Al-Enazy, H., Bolaji, Y., Hanjrah, S., Hussain, A., Makhlouf, S. \& Upshur, R. E. G. (2004). Psychosocial effects of SARS on hospital staff: Survey of a large tertiary care institution. CMAJ, 170(5), 793-798. doi:10.1503/cmaj.1031077

Qiu, J., Shen, B., Zhao, M., Wang, Z., Xie, B. \& Xu, Y. (2020). A nationwide survey of psychological distress among Chinese people in the COVID-19 epidemic: Implications and policy recommendations. General Psychiatry. BMJ Publishing Group, 1-3. doi:10.1136/gpsych2020-100213

Rossi, R., Socci, V., Talevi, D., Mensi, S., Niolu, C., Pacitti, F., Dimarco, A., Rossi, A., Siracusano, A. \& Lorenzo, G. D. (2020). COVID-19 pandemic and lockdown measures impact on mental health among the general population in Italy. MedRxiv, 1-12. doi:10.1101/2020.04.09.20057802

Rubin G James, Amlôt Richard, Page Lisa, Wessely Simon. Public perceptions, anxiety, and behaviour change in relation to the swine flu outbreak: cross sectional telephone survey BMJ 2009; 339 :b2651 
Shultz, J. M., Cooper, J. L., Wainberg, M. \& Neria, Y. (2016). The role of fear-related behaviors in the 2013-2016 West Africa Ebola virus disease outbreak. Current Psychiatry Reports. 1-15. doi:10.1007/s11920-016-0741-y

Sileo, K. M., Bogart, L. M., Wagner, G. J., Musoke, W., Naigino, R., Mukasa, B. \& Wanyenze, R. K. (2019). HIV fatalism and engagement in transactional sex among Ugandan fisherfolk living with HIV. Sahara J, Journal of Social Aspects of HIV/AIDS, 16(1), 1-9. doi:10.1080/17290376.2019.1572533

Sonderskov, K. M., Dinesen, P. T., Santini, Z. I. \& Ostergaard, S. D. (2020). The depressive state of Denmark during the COVID-19 pandemic. Acta Neuropsychiatrica. 226-228. doi:10.1017/neu.2020.15

Sun, L., Sun, Z., Wu, L., Zhu, Z., Zhang, F., Shang, Z., Jia, Y., Gu, J., Zhou, Y., Wang, Y., Liu, N. \& Liu, W. (2020). Prevalence and risk factors of acute Posttraumatic Stress Symptoms during the COVID-19 outbreak in Wuhan, China. MedRxiv, 1-17. doi:10.1101/2020.03.06.20032425

Tucci, V., Moukaddam, N., Meadows, J., Shah, S., Galwankar, S. C., \& Kapur, G. B. (2017). The forgotten plague: Psychiatric manifestations of Ebola, Zika, and emerging infectious diseases. Journal of Global Infectious Diseases, 9(4), 151-156. https://doi.org/10.4103/jgid.jgid_66_17

Vanderpool, R. C., Dressler, E. V. M., Stradtman, L. R. \& Crosby, R. A. (2015). Fatalistic beliefs and completion of the HPV vaccination series among a sample of young Appalachian Kentucky women. Journal of Rural Health, 31(2), 199-205. doi:10.1111/jrh.12102

Wang, C., Pan, R., Wan, X., Tan, Y., Xu, L., Ho, C. S. \& Ho, R. C. (2020). Immediate psychological responses and associated factors during the initial stage of the 2019 coronavirus 
disease (COVID-19) epidemic among the general population in China. International Journal of Environmental Research and Public Health, 17(5), 199-205. doi:10.3390/ijerph17051729

World Health Organization (2020). Rolling updates on Corona Virus as they happen. Retrieved from https://www.who.int/emergencies/diseases/novel-coronavirus-2019/events-as-they-happen 